\title{
LOS CAMINOS DEL CAMPO DE MONTIEL EN ÉPOCA DE CERVANTES
}

Es sin duda Don Quijote de la Mancha, novela de caminos y ventas, y salvo en dos ocasiones concretas, el Toboso y Barcelona, toda ella transcurre en los caminos y encrucijadas donde D. Quijote espera alcanzar la gloria y Sancho Panza su Ínsula.

A pesar de la insistencia de Cervantes que hasta cinco veces repite que las aventuras y desventuras de D. Quijote transcurren en el Campo de Montiel; en el prólogo; Cap. 1; Cap. VII; Cap. LII; y en el VII de la Segunda parte; ninguna de las rutas conocidas, siendo numerosas, se han trazado por los caminos del mismo.

Podríamos suponer segundas o torcidas intenciones, pero creo que esto es fruto exclusivo del desconocimiento por parte de quienes las trazaron, de esta comarca y sus caminos, alejada de todas partes, ignorada por muchos y mal estudiada por todos. Es por eso, que dentro de nuestras fuerzas, quisiera que ustedes conozcan los caminos del Campo de Montiel, donde según Cervantes, debemos buscar las huellas de Rocinante.

Mucho ha llovido para que se conserven, difícil resulta en una novela precisar con metro y reloj, como algunos quieren, las andanzas de nuestro héroe, pero parece indudable que si en algún sitio hemos de situarlas, ha de ser en estos caminos y parajes, que algo tendrán, cuando el genial Cervantes sitúa en ellos la acción de su novela.

Han sido numerosos los autores, Unamuno, Azorín, Jacacci, Galdós, y un largo etcétera, que han tratado de aprehender y descifrar los motivos de Cervantes para esta ubicación, motivos que si están claros en otras obras, nos son desconocidos en el Quijote, por eso, a ustedes, insignes filólogos y estudiosos de esta magna obra, 
lanzo el reto de lograrlo, y la invitación para que estando aquí no se marchen sin conocer esta comarca, la auténtica tierra de estas universales aventuras, y en la que D. Quijote pasaba por ser el más casto enamorado y más valiente caballero que muchos años a esta parte se vio en aquellos contornos.

Tampoco debe resultarnos extraña la expresión «y comenzó a caminar por el antiguo y conocido Campo de Montiel», ya que desde el sur se accede al mismo por un camino que todavía en el M. T. N. de 1888, figura con el nombre de vía Aníbal.

No obstante, en la última y al parecer completa edición del Quijote de Francisco Rico, ya se incluye un mapa del Campo de Montiel de 1575, y otro con uno de sus caminos principales, el de Cuenca a Granada, así como una litografía de Venta Nueva, pórtico de Sierra Morena desde el norte; con lo que podríamos entender que esta comarca empieza a conocerse y considerarse como la única patria de D. Quijote.

Centrándome en el tema de mi comunicación, pues el amor a mi tierra me llevaría a divagaciones interminables, empezaré con unas palabras de Corchado Soriano: «El nudo de comunicaciones antiguas que forma el Campo de Montiel, con ramales hacia Andalucía, Campos de Calatrava y San Juan, Uclés y Cuenca, y el Levante y Sudeste, hizo que, indudablemente, la resistencia musulmana se aferrara a él y no pudo ser desalojada hasta que, desbordada por ambos lados, su permanencia les fue insostenible, pero su apego a esta región y el valor que le concedían, se demuestra evidentemente con ser el único punto de la meseta donde los musulmanes volvieron a poner el pie mucho después de su reconquista». Red de comunicaciones que, en palabras del mismo autor, estuvo vigente hasta bien entrado el siglo XVII.

Sería el descubrimiento de América y traslado de mercancías a los puertos atlánticos lo que determinaría la pérdida de importancia de éste, y de los caminos que a él conducían, que venían siendo usados desde la más remota antigüedad, y de los que ya Tito Livio nos dice que por el entonces llamado camino de Aníbal se llevó Hércules los ganados del rey Gerión de ahí su primer nombre de Vía Heraclea.

Esta situación ventajosa la da el puerto por donde se atraviesa Sierra Morena, que es en longitud y pendiente, como el diez por ciento de Despeñaperros; hasta el punto de que el primer proyecto de ferrocarril Madrid-Sevilla, se hizo por aquí, aunque por otras razones se hizo por paso tan ilógico, que el actual trazado del AVE ha evitado.

Del mismo modo el Campo de Montiel era paso obligado a Murcia y Cartagena, por eso, Alcaraz, ciudad limítrofe al mismo, 
lleva en su escudo la leyenda "Llave de España», por encontrarse entre Toledo, Murcia, Granada, Jaén y La Mancha, en palabras de D. Pascual Madoz.

Sierra Morena, límite sur del Campo de Montiel, termina aquí para coincidir perpendicularmente con el Sistema Prebético en las sierras de Alcaraz y Segura perdiendo ya su abruptuosidad y permitiendo un paso más llano, por lo que en época de tracción animal, con el puerto de Cartagena y la ciudad de Granada, la tercera en población, desempeñaron estos caminos y comarca un papel importante en la historia y comercio de España.

De muy diversas formas se ha llamado a este puerto a lo lago de la historia, como Saltus Castulonensis, lo citan los romanos por su proximidad a Castulo, actual Linares; en la Edad Media se le llama puerto de Montizón, por tener al norte el castillo de este nombre y al sur las aldeas de Montizón, así lo llama Sancho IV en una carta de privilegios concedida a Santisteban del Puerto para la custodia del mismo.

Mon-tizón, es decir, montes negros, como también lo llama Cervantes en los epitafios de los Académicos de la Argamasilla, haciendo andar a D. Quijote el Campo de Montiel desde la gran sierra Negra hasta el herboso llano de Aranjuez.

También se llamó puerto de San Esteban, tal vez por la proximidad a Santisteban del Puerto, o viceversa, Madoz, y en la comarca, lo llama Barranco Hondo.

$\mathrm{Al}$ ser la comunicación natural entre el Levante y la Bética, para algunos autores sobre este camino se desarrolló la segunda guerra púnica, acampando en invierno el ejército cartaginés en las proximidades del mismo para impedir el paso a la Bética de los romanos; conservándose todavía un lugar «Piedras Negras» con el mismo nombre, y donde en el año 210 a. C. Asdrúbal burla a Claudio Nerón con una estratagema que luego usaría César en la guerra de las Galias.

Algunos autores sitúan en esta comarca la primera batalla de Munda, al encontrarse el ejército romano bajando por la Vía Augusta, y el cartaginés viniendo por el de Cartagena; cruce de caminos que Cervantes conocería cuando hace decir al cura Pero Pérez, a la princesa Micomicona, subiendo de Sierra Morena, «por mitad de mi pueblo hemos de pasar, y allí podrá tomar la derrota de Cartagena».

Pues bien, el camino por excelencia del Campo de Montiel (núm. 1) es el que atravesándolo de sur a norte, entra por el citado puerto, y también ha cambiado de nombre a lo largo de la historia; es el camino de Hércules o vía Heraclea, camino de Aníbal, y después de los arreglos del mismo en época romana, vía Augusta, 
llamado más tarde por los musulmanes camino de los arrecifes, es decir, camino empedrado.

Hoy en la zona se le conoce como camino real, y en el M. T. N. lo encontrarán ustedes como camino o vereda de los Serranos, también llamado Cañada Real de Andalucía, pues como casi la mayoría, también hacía de vía pecuaria, siendo usado hasta la desaparición de la trashumancia.

Figura en los M. N. T. de 1888 como vía de Aníbal entre las Aldeas de Montizón y Puebla del Príncipe; y como vía romana entre Villanueva de la Fuente y Viveros.

Fue la primera calzada que hicieron los romanos y unía las columnas de Hércules en Cádiz, con Roma, y su documentación es clara y abundante, ya Plinio la refleja en su mapa de caminos de Hispania; los vasos Apolinares o de Vicarello, para esta zona dan las siguientes mansiones: Ad Morum y Solaria en Jaén, las actuales Navas de San Juan y Aldeas de Montizón, al sur de Sierra Morena; Mariana, y Mentesa ya en el Campo de Montiel, las actuales ermita de Mairena en Puebla del Príncipe y Villanueva de la Fuente, ciudad esta que Ptolomeo ya sitúa correctamente en latitud y longitud; Libisossa, ya en Albacete, la actual Lezuza, de donde por Chinchilla, Almansa y Játiva se dirigía a Tarragona.

Lezuza, a unos 30 kilómetros de la cueva de Montesinos, hace que este camino sea el que más probablemente llevó D. Quijote hasta Barcelona.

También citan dicho camino Tito Livio en las guerras Púnicas; Petronio en el Satiricón; Polión en su Epistolario Ciceroniano, haciendo estos últimos referencia al bandolerismo existente que interceptaba los correos de Roma, situación endémica que siglos más tarde querría solucionar nuestro buen Hidalgo.

Encarga Roma a Sixto Mario, la normalización de este paso, personaje que pasaba por ser el más rico del Imperio, tanto que acusado falsamente de incesto y arrojado de la peña Tarpeya, sus bienes son confiscados por el propio emperador; su negocio era la explotación de las minas de Hispania y de su nombre Mario, algunos hacen derivar el de Sierra Mairena o Morena.

Fue él quien convirtió las paradas o ventas de la comarca en poblaciones y castillos, de los que hay sólo en la parte norte, hasta 32, y por eso leemos en las relaciones topográficas de algunos de estos pueblos como Montiel, que en su término no hay ventas por estar todos los pueblos a menos de dos leguas, siendo en una de estas torres del sur donde murió Cneo Escipión.

A través de esta vía se puede documentar el paso de Tariq, Abderramán III con su ejército, Alfonso VIII en la batalla de las Navas, según algunos autores, el Cid, para entrevistarse con Alfon- 
so VI que sitiaba Úbeda, Enrique II, Santa Teresa, Cosme de Médicis, el pícaro D. Gregorio Guadaña y Felipe IV en su viaje a Doñana, viaje que nos relata Quevedo en 1624 en carta al marqués de Velada.

Figura igualmente en los repertorios de Villuga y Meneses, Itinerario XIV, y en la Cosmografía de Fernando Colón como itinerario de Barcelona a Sevilla.

Naturalmente que a este puerto afluyen otros caminos aprovechando su orografía, y encontramos al este el camino Real del Ojuelo (núm. 2), que desde la sierra de Alcaraz y venta del Ojuelo, próxima a Puebla del Príncipe, llega a Venta Nueva.

Como ramal del primero y desde Puebla del Príncipe hasta Villanueva de la Fuente, encontramos en el M. T. N. un camino real de Levante a Andalucía, que pasa al este y por debajo de Albaladejo, nombre ya muy caminero.

$\mathrm{Al}$ oeste de la vía Augusta y desde el norte, encontramos el camino de Cuenca a Granada (núm. 3), que desde Villarrobledo, Ossa de Montiel, Villahermosa, Montiel y Villamanrique llega a Venta Nueva, figura en los repertorios de Meneses y muy probablemente sea el tan buscado camino de los Berones, conservándose tramos empedrados en Montiel y Villahermosa.

Por este camino pasa hacia el sur el 20 de septiembre de 1836 el general carlista Gómez, documentándose así con exactitud el mismo.

Más al oeste del citado (núm. 4) encontramos el que desde Venta Quesada, por Membrilla, La Solana, Alcubillas y Cózar llega a Venta Nueva, que cruza el río Jabalón en Alcubillas por un puente que Madoz llama de la carretera vieja de Andalucía, camino Real de carros de Andalucía, según Corchado Soriano; que fue el seguido desde Madrid por Felipe IV en 1624, lo que nos hace suponer que estuvo en servicio al menos hasta la apertura de Despeñaperros en 1772 .

En la citada Venta Quesada, sitúa Richard Ford en 1845, la venta donde D. Quijote es armado Caballero.

Según los itinerarios de Antonino, del Campo Laminitano, situado en esta región, partían la vía 29 (núm. 5), que enlazaba Mérida a Cartagena, pasando por Sisapo y atravesando el Campo de Montiel de oeste a este, y cruzándose con la vía Augusta en perpendicular en Villanueva de la Fuente; camino hoy seguido por las carreteras Badajoz-Ciudad Real y Ciudad Real-Murcia, separándose del mismo un ramal en Cañamares, llamado todavía camino de Alicante.

Atravesaba el Valle de Alcudia por Sisapo, La Bienvenida actual, y llega al Campo de Montiel por San Carlos del Valle como camino de las carretas y al norte de Villanueva de los Infantes como 
camino real de la Plata, como podemos ver en M. T. N. actual, y del que dice Fernando Colón en el siglo XVI que estaba empedrado.

Ya que la plata del valle de Alcudia se transportaba en carretas hasta Cartagena para su embarque a Cartago y Roma, parecen lógicos estos nombres, y debió ser el camino más importante de Villanueva de los Infantes cuando en él todavía se conserva la cruz de los asaeteados por la Santa Hermandad.

Más al norte y casi paralelo al anterior nos encontramos con el camino de Ciudad Real a Alicante (núm.6), hoy traducido por la carretera 430 de Ciudad Real a Valencia que atraviesa el Campo de Montiel por Membrilla-La Solana-Alhambra-Ossa de Montiel y pasa cerca de Lezuza; éste además pasaba por las Lagunas de Ruidera cerca de la cueva de Montesinos y ermita de San Pedro.

Por último, también partía del Campo Laminitano la vía 30 de los Itinerarios de Antonino, que lo unía con Toledo, y que partiendo de Villanueva de la Fuente pasa por Cañamares, las Lagunas de Ruidera donde se cruza por debajo de la ermita de San Pedro con el anterior, sigue por Argamasilla y Consuegra a Toledo.

Este camino todavía se conoce y así figura en el M. N. T. como camino de Alandumbar, nombre que según el hispanista argelino Ahmed Abi-Ayad, podríamos traducir como el camino por donde Dios soluciona todo.

Naturalmente que todos los caminos descritos tenían un sinfín de atajos y desviaciones que se usaban según la estación seca o lluviosa, que en unos casos eran intransitables, o más agradables en verano por el agua y la vegetación.

Vemos cómo se forma una red de caminos y encrucijadas en un terreno casi desértico como Cervantes y Quevedo califican a esta zona, que al no estar y menos en ese tiempo cultivada, los atajos y rodeos son innumerables resultando fácil perderse, especialmente en el camino de Alandumbar, por lo que también parece lógico este nombre musulmán.

No sería pues aventurado pensar que para una novela de caminos y encrucijadas le pareciese a Cervantes el Campo de Montiel, el mejor lugar donde ubicar las aventuras de su protagonista, pues sabemos por Cristóbal Pérez Pastor en su documento LXVIII del 11 tomo, pág. 241, que Cervantes en el año 1593 hace un recaudo en Mairenilla, y el 26 de enero de 1594, otro en Villamanrique de quince fanegas y media de trigo y cinco de cebada; documento incompleto y con el que tampoco tiene suerte el Campo de Montiel.

No obstante mucho tenía que conocer Cervantes esta comarca ya que los datos geográficos o camineros que nos da en El Quijote son tan precisos que no admiten duda; así en el Cap. XXIII nos dice 
después de haber salido de la cueva de Montesinos «no lejos de aquí, está una ermita que tiene una habitación o pequeña casa».

Sitúa la ermita cercana y por debajo de la cueva, ermita que aún persiste aunque reconstruida, con la casa del santero al lado y una habitación que hacía de posada, donde se encuentran con un mozo que va a Cartagena, donde se armaban Galeras, y ellos camino de Barcelona, siendo este el punto justo donde se cruzan los dos caminos como hemos visto.

Esta habitación o pequeña casa, que es la única de segura localización en toda la obra, está lamentablemente dejada de los poderes públicos, aunque restaurada, suponemos que bien, por los propietarios.

Si en esta comarca y sus caminos podemos ajustar las distancias que Cervantes da, y encontrar los detalles que cita; si además repite hasta cinco veces que es el Campo de Montiel por donde Don Quijote corre sus aventuras, parece indudable que aquí es donde habrá que situar la ruta que algún día se haga de las mismas.

No sé con certeza los fines de la Asociación de Cervantistas, si su campo es exclusivamente el estudio filológico de la obra de Cervantes, o si tiene otros ámbitos de actuación, pero en nombre de la Asociación de Amigos del Campo de Montiel, someto a su consideración otras posibilidades que sin duda llevarían a la mayor difusión y mejor conocimiento de la misma.

Son proyectos ambiciosos pero realizables, si se cuenta con el suficiente empeño e ilusión; amén de que habiendo una ruta conocida y verosímil sería visitada, se podría hacer en la comarca una especie de aula abierta donde con las escenificaciones oportunas se mostrasen las nobles ideas de Cervantes puestas en boca de Don Quijote, como ejemplos de sus sentimientos de igualdad, justicia, heroicidad, etc., virtudes que me atrevería a calificar de precursoras de los derechos humanos.

Puestos a soñar, también se podría hacer, como expuse en Menorca, vaciando la cueva de Montesinos, una escenificación fija del sueño de Don Quijote dentro de la misma. Bueno, ya advertí al principio que mi ilusión por mi tierra me llevaría como paisano de Don Quijote a pensar, decir y proponer quijotadas: nosotros trabajaremos para conseguirlo.

JUSTINIANO RODRÍGUEZ CASTILlO 


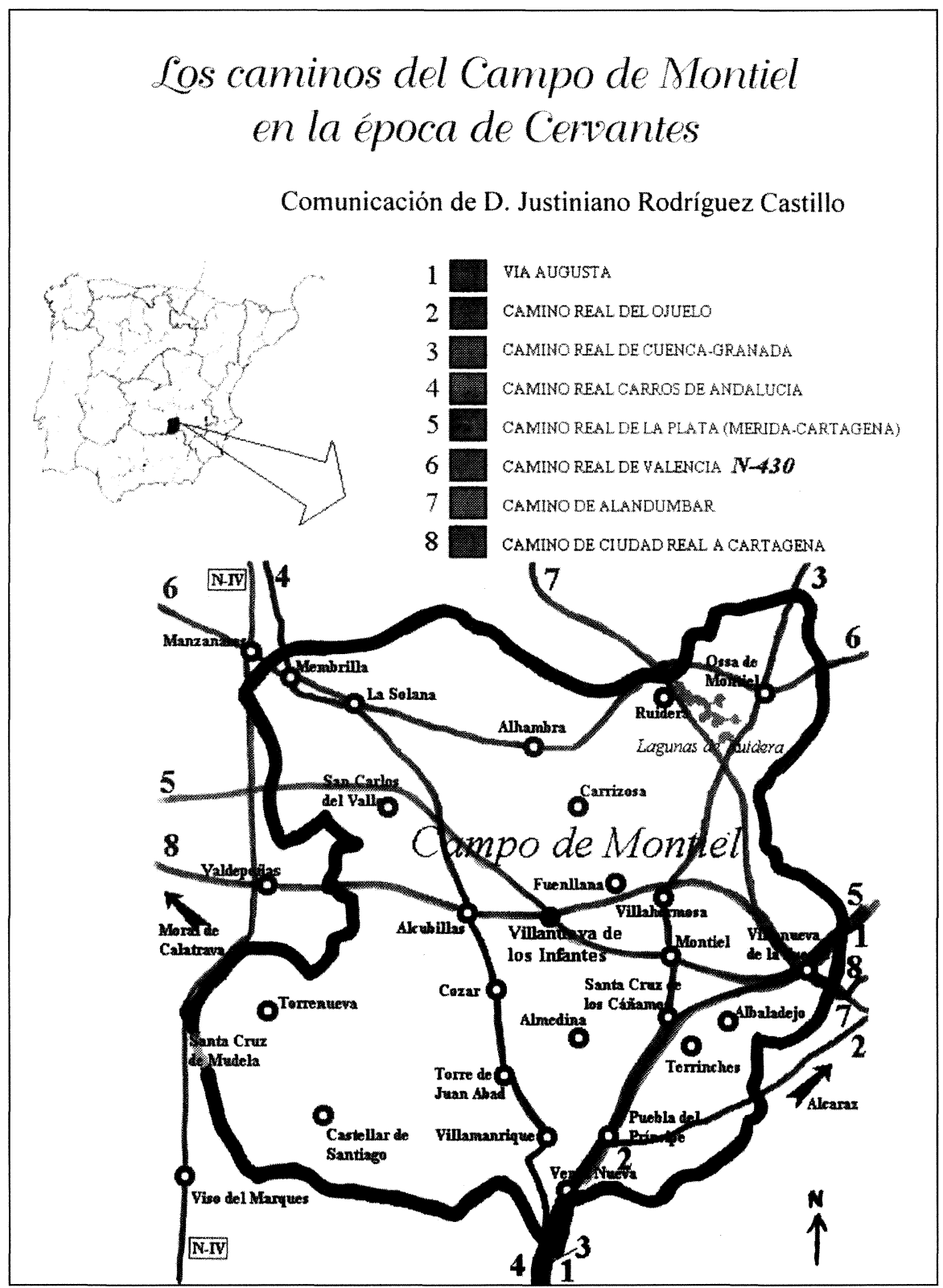

